466

USE OF A 27-GENE IMMUNO-ONCOLOGY (IO) ASSAY TO ASSOCIATE RESPONSE TO SINGLE-AGENT IMMUNE CHECKPOINT INHIBITOR (ICI) THERAPY IN ADVANCEDSTAGE NSCLC PATIENTS FROM A LARGE CANADIAN COHORT

${ }^{1}$ David Saltman*, ${ }^{2}$ Nicole Croteau, ${ }^{1}$ Heather Lockyer, ${ }^{3}$ Rob Seitz, ${ }^{3}$ Frank McMahon, ${ }^{3}$ Jeremy Spille, ${ }^{3}$ Andrea Dickey, ${ }^{3}$ Matthew Varga, ${ }^{3}$ Kim McGregor, ${ }^{3}$ Tyler Nielsen, ${ }^{3}$ David Hout, ${ }^{3}$ Brock Schweitzer, ${ }^{3}$ Douglas Ross, ${ }^{4}$ David Gandara. ${ }^{1} B C$ Cancer, Victoria, Canada; ${ }^{2}$ The University of British Columbia, Victoria, Canada; ${ }^{3}$ Oncocyte Corporation, Nashville, TN, USA; ${ }^{4}$ UC Davis, Sacramento, CA, USA

Background Lung cancer is the leading cause of cancer-related deaths worldwide. The advent of ICIs specifically targeting programmed cell death protein-1 (PD-1), or its ligand (PD-L1) represents a major therapeutic advance that is now included in standard of care regimens for non-small-cell-lung cancer (NSCLC). PD-L1 expression measured by immunohistochemistry (IHC) staining is the current gold standard predictive biomarker for immune checkpoint inhibitor (ICI) therapy in NSCLC, however many factors beyond PD-L1 expression alone affect the outcome of ICI therapy. Evaluation of other factors to better inform clinical practice will reduce both the potential for adverse immune-related toxicities and expenditure on ineffective costly therapies while potentially identifying patients otherwise missed by PD-L1 staining. The 27-gene IO assay is a RT-qPCR based gene expression panel ${ }^{1}$ that was developed to classify the tumor immune microenvironment (TIME). It has been shown to be associated with response to ICI therapy in multiple tumor types including triple negative breast cancer, metastatic urothelial carcinoma, and NSCLC where the association was independent of PD-L1 status in patients treated either with monotherapy or combination therapy. ${ }^{2}$ Currently, BC Cancer measures PD-L1 status by IHC using the PD-L1 22C3 PharmDx assay and reports the tumor proportional score (TPS) to inform clinical decision. Patients with a TPS $\geq 50 \%$ may be eligible for first-line treatment with ICI monotherapy and those with $<50 \%$ TPS are eligible for second line or later ICI monotherapy. We established this retrospective study of ICI monotherapy treated NSCLC patients to assess the 27-gene IO assay as an informative biomarker for NSCLC ICI treatment decisions.

Methods This retrospective study is utilizing the BC Cancer Study Database to select approximately 150 patients with stage IIIB or IV NSCLC treated with single-agent ICI therapy across four BC Cancer centers from 2017 forward (figure 1). Patients are selected based on availability of adequate biopsy specimens (FFPE with at least 20\% tumor content), availability of PD-L1 IHC results or sufficient tissue to conduct staining, and for whom outcome data is available via chart review. RNA from patient samples is isolated from FFPE biopsies (either primary or metastatic sites) and those that yield $\geq 50$ ng RNA will be analyzed by the 27 -gene IO assay ${ }^{1}$ to derive IO scores (IO positive or IO negative) based on previously defined thresholds. ${ }^{3}$ The association between patient outcomes on ICI monotherapy and IO scores and PD-L1 IHC will be reported and compared.

\section{BC Cancer}

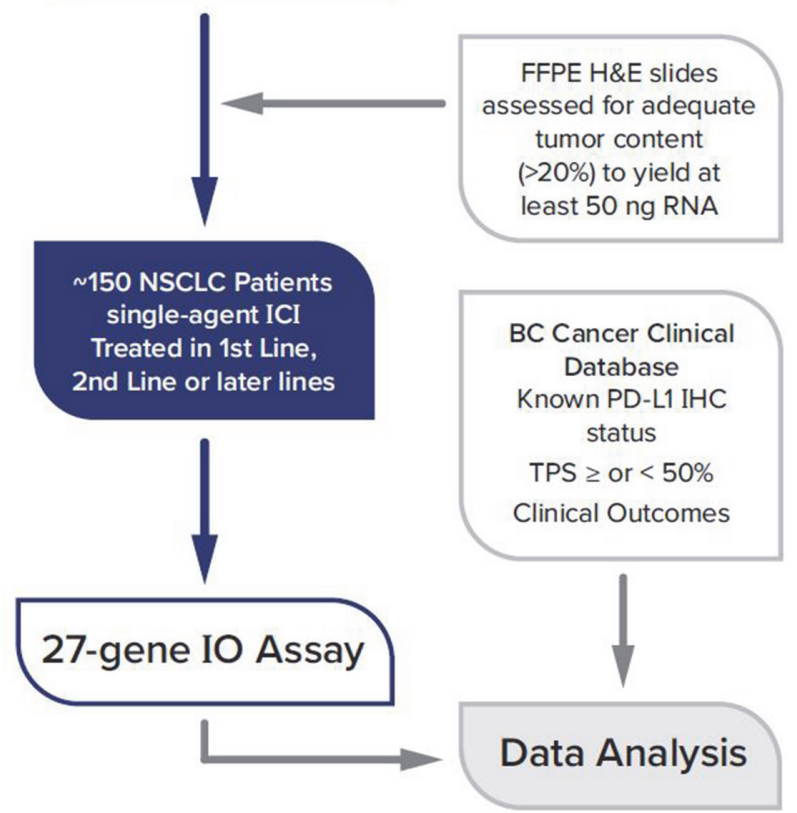

Abstract 466 Figure 1 Schematic representation of patient workflow for

\section{REFERENCES}

1. Saltman, A, et al. Prostate cancer biomarkers and multiparametric MRI: is there a role for both in prostate cancer management? Ther Adv Urol 2021;13: 1756287221997186.

2. Ranganath HJA, Smith JR, et al. One-year progression-free survival in lung cancer patients treated with immune checkpoint inhibitors is significantly associated with a novel immunomodulatory signature but not PD-L1 staining. in SITC. Journal Immunotherapy Cancer. 2019.

3. Nielsen, TJ, et al. A novel immuno-oncology algorithm measuring tumor microenvironment to predict response to immunotherapies. Heliyon 2021;7(3):e06438.

Ethics Approval The University of British Columba BC Cancer Research Ethics Board Chair, Vice-Chair or second Vice-Chair, has reviewed the above described research project, including associated documentation, and finds the research project acceptable on ethical grounds for research involving human subjects. All participants have provided informed consent before taking part in the study. REB Number H20-02635.

http://dx.doi.org/10.1136/jitc-2021-SITC2021.466 